\title{
The use of vasodilator myocardial perfusion imaging in severe aortic stenosis: Is it time for a new prospective study?
}

\author{
Ioannis Parastatidis, $\mathrm{MD}, \mathrm{PhD},{ }^{\mathrm{a}}$ and Stamatios Lerakis, $\mathrm{MD}^{\mathrm{a}}$ \\ ${ }^{a}$ Division of Cardiology, Department of Medicine, Emory University School of Medicine, \\ Atlanta, GA
}

Received Mar 3, 2016; accepted Mar 4, 2016

doi: 10.1007/s12350-016-0468-5

\section{See related editorial, pp. 1200-1213}

Aortic stenosis often coexists with coronary atherosclerotic disease, and with the exception of men less than 40 years old and premenopausal women, part of the workup in clinical practice includes assessment for coronary disease. Conversely, the presence of angina in patients with aortic stenosis is less specific for coronary obstructive disease, since chest pain could be a result of increased myocardial wall stress, increased oxygen demand, or subendocardial ischemia. According to the guidelines, ischemic workup should be preferably performed using coronary angiography, since exercise stress testing has been traditionally considered a contraindication for symptomatic ${ }^{1}$ and even asymptomatic ${ }^{2}$ severe aortic stenosis. Vasodilator stress single photon emission computed tomography (SPECT) or positron emission tomography (PET) imaging could also provide valuable information about the presence of coronary ischemia in this patient population; however, the acute systemic vasodilatory effect of adenosine, dipyridamole, and regadenoson has been rightfully causing disquiet among this population. Therefore, the safety, and the diagnostic and prognostic significances of vasodilator stress imaging have not been well studied in aortic stenosis and are not well established.

In the last decade, the development of minimally invasive and percutaneous approaches for the treatment

Reprint requests: Stamatios Lerakis, MD, Division of Cardiology, Department of Medicine, Emory University School of Medicine, 1365 Clifton Road NE, Suite AT503, Atlanta, GA 30322; sleraki@emory.edu

J Nucl Cardiol 2017;24:1200-13.

1071-3581/ $\$ 34.00$

Copyright (c) 2016 American Society of Nuclear Cardiology. of aortic stenosis resulted in the consideration for aortic valve replacement of significantly older patients and patients that would have previously deemed inoperable. Not surprisingly, these patients frequently have coronary atherosclerotic lesions, which need further ischemic assessment. Therefore, vasodilator stress imaging can be a valuable tool for the clinician in order to evaluate the ischemic burden and guide the revascularization strategy.

In this issue of the journal, Hussain et al examined retrospectively the safety of vasodilator stress imaging in a population with severe aortic stenosis. A total of 95 patients with a mean aortic valve area of $0.79 \mathrm{~cm}^{2}$ calculated using the continuity equation received vasodilator stress imaging; 24 patients received adenosine, 29 dipyridamole, and 43 regadenoson infusion. In general, the use of vasodilator stress agents in this population was tolerated well; $17 \%$ of the patients reported dyspnea and $7 \%$ reported headache as the most frequent side effects. Interestingly, no sustained supraventricular, ventricular cardiac arrhythmia, or high-degree AV block was reported. The authors found that the use of vasodilator was associated with a significant, but asymptomatic, decrease in the systolic blood pressure. Specifically, the systolic blood pressure decreased by $17 \pm 20 \mathrm{~mm} \mathrm{Hg}$ in aortic stenosis vs $7 \pm 18 \mathrm{~mm} \mathrm{Hg}$ in the age-and sex-matched control group. The difference was statistically significant for the group that received adenosine, and there was a trend toward a larger drop in the systolic blood pressure in the dipyridamole and regadenoson groups compared to the control population. Interestingly, symptomatic hypotension was not noted in any of the patients. The findings of this study are consistent with the prior reports, ${ }^{3}-{ }^{7}$ which found that about $20 \%$ of individuals with severe aortic stenosis that underwent vasodilator stress test developed significant hypotension, and only the minority of those became symptomatic. These 
studies were elegantly summarized recently ${ }^{2}$ and by the authors. The findings of this study agree with the previous reports and suggest that vasodilators are well tolerated and can be used safely in patients with severe aortic stenosis.

It is now becoming apparent that both cardioselective (regadenoson) and possibly non-selective (adenosine and dipyridamole) vasodilators are well tolerated by patients with severe aortic stenosis and the ischemic burden can be safely and accurately evaluated using non-invasive stress testing. In addition, the cardiac computed tomography, which is acquired in the majority of the cases for transcatheter aortic valve replacement (TAVR) planning, can visualize well at least the proximal segments of the coronary tree. Therefore, with the combination of vasodilator SPECT or PET imaging and cardiac computed tomography, we can achieve an accurate non-invasive assessment of the coronaries and proceed with cardiac catheterization only in patients with significant ischemic burden, which is estimated to be less than $50 \%$ of the patients.

Although this work helps significantly to further our understanding of the hemodynamic effects of vasodilators in severe aortic stenosis, it also generates questions than can probably be answered with further studies. Firstly, it would be interesting to examine what percentage of this population suffered from severe symptomatic aortic stenosis, and whether the vasodilator challenge is equally well tolerated in subjects with symptomatic vs. asymptomatic aortic stenosis. We believe that this is of critical importance, because this group of patients probably benefits the most from aortic valve replacement. Secondly, it would also be interesting to know whether an exaggerated hemodynamic response to vasodilator challenge is associated with poor short or longer term outcomes (such as mortality, hospitalizations, stroke, myocardial infarction, heart failure, syncope). Thirdly, since high-degree AV block and need for permanent pacemaker insertion is a relatively frequent complication after percutaneous aortic valve replacement, it is worth testing whether transient AV block, bundle branch block, or arrhythmia induced by vasodilator challenge can predict which patient will require permanent pacing after TAVR. Finally, we believe that the use of vasodilator stress testing in severe aortic stenosis cannot be comprehensively evaluated without studying the peri-procedural and early postprocedural outcomes in patients who underwent TAVR without prior coronary angiography, solely based on reassuring non-invasive ischemic assessment. We recognize that this is not the standard clinical practice at present, but studies like the one reported in this issue of the journal by Houssain et al can establish the foundation, based on which non-invasive imaging could be tested prospectively against coronary angiography for the pre-procedural evaluation of severe aortic stenosis.

\section{Disclosure}

The authors have indicated that they have no financial conflict of interest.

\section{References}

1. Nishimura RA, Otto CM, Bonow RO, Carabello BA, Erwin JP 3rd, Guyton RA, et al. 2014 AHA/ACC guideline for the management of patients with valvular heart disease: A report of the American College of Cardiology/American Heart Association Task Force on Practice Guidelines. J Am Coll Cardiol 2014;63:e57-185.

2. Henzlova MJ, Duvall WL, Einstein AJ, Travin MI, Verberne HJ. ASNC imaging guidelines for SPECT nuclear cardiology procedures: Stress, protocols, and tracers. J Nucl Cardiol 2016. doi: 10.1007/s12350-015-0387-x.

3. Cremer PC, Khalaf S, Lou J, Rodriguez L, Cerqueira MD, Jaber WA. Stress positron emission tomography is safe and can guide coronary revascularization in high-risk patients being considered for transcatheter aortic valve replacement. J Nucl Cardiol 2014;21: 1001-10.

4. Patsilinakos SP, Kranidis AI, Antonelis IP, Filippatos G, Houssianakou IK, Zamanis NI, et al. Detection of coronary artery disease in patients with severe aortic stenosis with noninvasive methods. Angiology 1999;50:309-17.

5. Burgstahler C, Kunze M, Gawaz MP, Rasche V, Wohrle J, Hombach $\mathrm{V}$, et al. Adenosine stress first pass perfusion for the detection of coronary artery disease in patients with aortic stenosis: A feasibility study. Int J Cardiovasc Imaging 2008;24:195-200.

6. Avakian SD, Grinberg M, Meneguetti JC, Ramires JA, Mansur AP. SPECT dipyridamole scintigraphy for detecting coronary artery disease in patients with isolated severe aortic stenosis. Int J Cardiol 2001;81:21-7.

7. Demirkol MO, Yaymaci B, Debes H, Basaran Y, Turan F. Dipyridamole myocardial perfusion tomography in patients with severe aortic stenosis. Cardiology 2002;97:37-42. 